\title{
Surface Plasmon Resonance sensor based on a D-shaped photonic crystal fiber with a bimetallic layer
}

\author{
Markos P. Cardoso ${ }^{1, *}$, Anderson O. Silva ${ }^{2}$, Amanda F. Romeiro ${ }^{1}$, M. Thereza R. Giraldi ${ }^{3}$, João C.W.A. Costa ${ }^{1, * *}$, \\ José. L. Santos ${ }^{5,6}$ José. M. Baptista ${ }^{4,6}$ and Ariel Guerreiro ${ }^{5,6}$ \\ ${ }^{1}$ Federal University of Pará, Applied Electromagnetism Laboratory, Rua Augusto Corrêa, 01, 66075-110, Belém, Pará, Brazil \\ ${ }^{2}$ Federal Center for Technological Education Celso Suckow da Fonseca, Av Maracana, 20271-110 - Rio de Janeiro, Brazil \\ ${ }^{3}$ Military Institute of Engineering, Laboratory of Photonics, Praça Gen. Tibúrcio, 80,22290-270, Rio de Janeiro, Brazil \\ ${ }^{4}$ Faculdade de Ciências Exatas e da Engenharia, Univerisidade da Madeira, Campus da Penteada, 9020-105 Funchal, Portugal \\ ${ }_{5}^{5}$ Faculadade de Ciências da Universidade do Porto, Rua do Campo Alegre s/n, 4169-007 Porto, Portugal \\ ${ }^{6}$ INESC TEC, Rua Dr Roberto Frias, 4200-465 Porto, Portugal
}

\begin{abstract}
The investigation of a D-shaped photonic crystal fiber sensor with a bimetallic layer for operation at the visible and infrared spectra is presented. The bimetallic layer is composed by silver and gold slabs deposited adjacently on the flat face of the fiber. It is shown that this architecture allows the excitation of two sharply distinguished plasmon resonance, which suggest potential applications for multiparameter sensing.
\end{abstract}

\section{Introduction}

Surface plasmon resonance (SPR) sensors have attracted much attention due to their technical features as highly sensitive characteristic and wide operating spectral range [1]. Sensors based on SPR have many potential applications such as biological detection [2]. Owing to advantages such as low loss and large mode area, photonic crystal fibers (PCF) have been used as a suitable platform for exciting surface plasmon resonances. Besides, guiding modes can be easily manipulated by varying the PCF's structural parameters [3]. A large variety of SPR sensors constructed with PCFs have been reported as: refractive index sensors [1], temperature sensors [4] and chemical sensors [5].

A sensing platform based on a D-shaped photonic crystal fiber with a bimetallic layer is theoretically investigated in this work. This is a new approach of a multiplasmonic sensing configuration, that is not based on coupling between plasmons in overlaid metallic films. Rather, it considers distinct and independent plasmons, with different responses that may facilitate simultaneous measurements of more than one physical parameter, as demonstrated for another design in Ref. [6]. In this study we consider a simpler design, with a bimetallic layer formed by two adjacent slabs of silver and gold, deposited on the flat surface of the PCF. Numerical computations have shown that a sensitivity of $3010 \mathrm{~nm} / \mathrm{RIU}$ is achieved for the measurement of a single parameter (refractive index).

\section{Designed Structure}

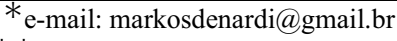

**e-mail: jweyl@ufpa.br
}

The scheme of the structure proposed is depicted in Figure 1. It is obtained by a side polished PCF with a hexagonal array of air holes on a matrix of fused silica.

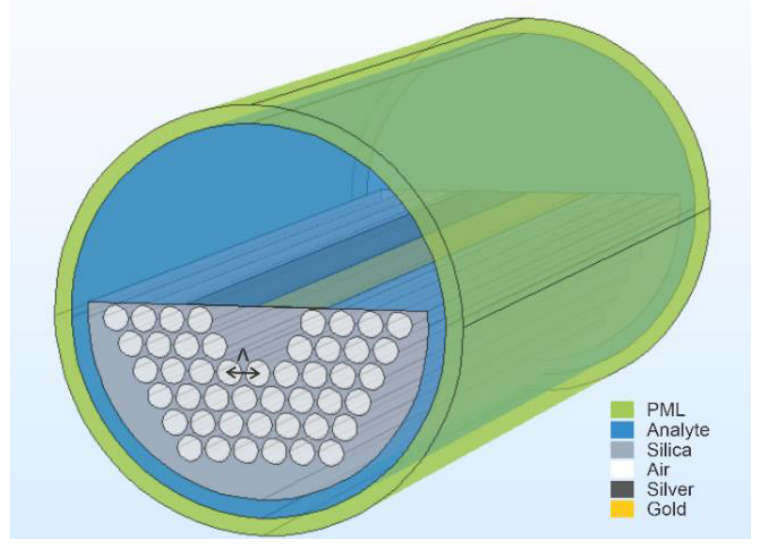

Figure 1. Schematic design of the D-shaped photonic crystal fiber with a bimetallic layer.

The diameter of the fiber is $\mathrm{D}=24 \mu \mathrm{m}$ and the diameters of the air holes is designated by $\mathrm{d}$. The distance between two adjacent air holes is kept fixed at $\Lambda=2 \mu \mathrm{m}$ while the ratio among $\mathrm{d}$ and $\Lambda$ is $\mathrm{d} / \Lambda=0.88$. The Finite Element based software COMSOL Multiphysics [7] was used to carry out numerical calculation. To adequately evaluate the sensing properties of the $\mathrm{D}$-shaped fiber, we applied a perfectly matched layer (PML) boundary condition with $10 \%$ of the fiber diameter for the truncation of the computational domain. 


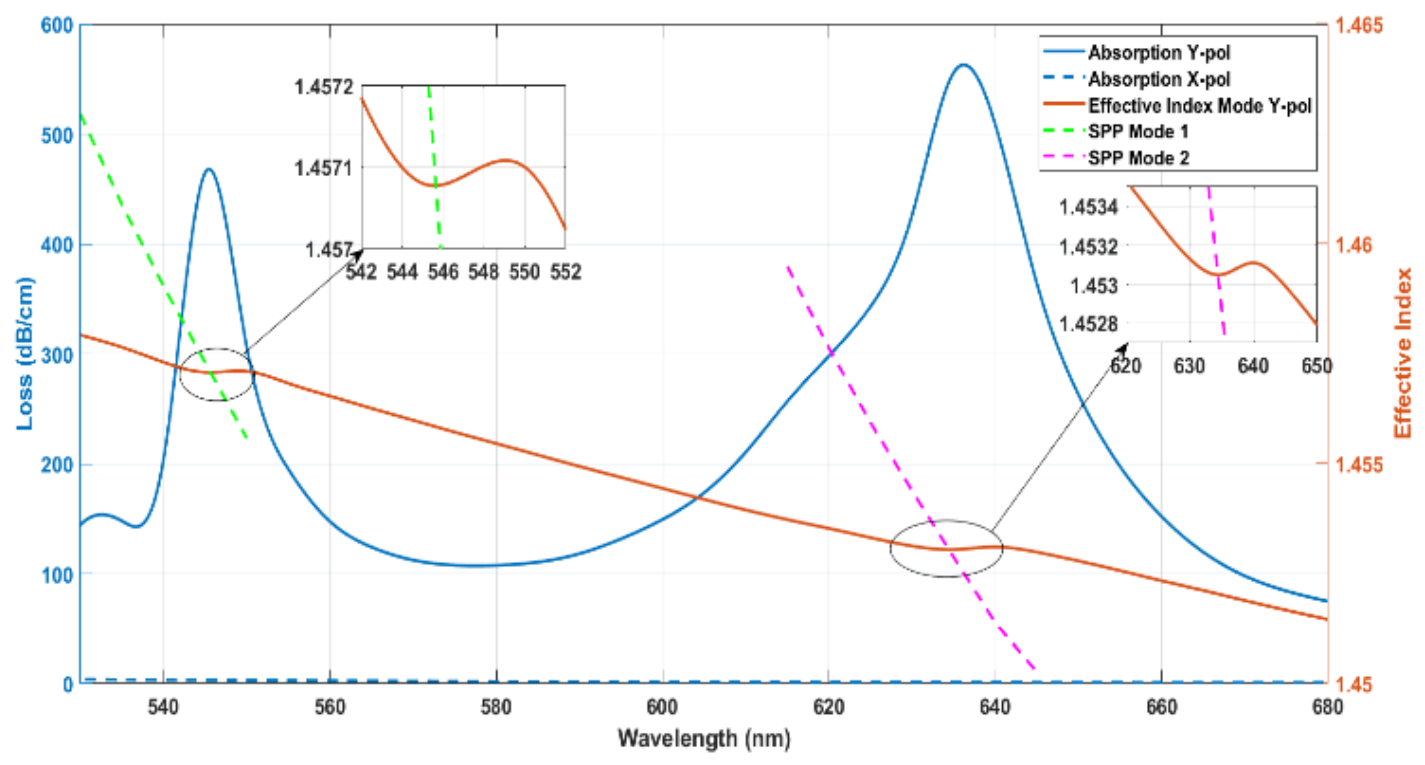

Figure 2. Dispersion and absorption curves of the D-shaped PCF with a bimetallic layer.

\section{Results and Discussion}

The dispersion curves for the SPP modes and the fundamental fiber mode together with the absorption curve are plotted in Figure 2 for $n_{\text {analyte }}=1.36$. The resonant coupling is reached at the phase matching between the SPP mode and the fiber guided mode, resulting in maximum confinement losses. The computed sensitivity is accounted for the variation of the resonant wavelength of maximum absorption for a variation of $10^{-2}$ in the refractive index of the analyte. Preliminary results are presented in Table 1, where a sensitivity of $3010 \mathrm{~nm} / \mathrm{RIU}$ and resolution of $3.32 \times 10^{-5}$ RIU are achieved considering an experimental spectral variation detection of $0.1 \mathrm{~nm}$.

Table 1. Sensitivity and Resolution for the variation of analyte refractive index.

\begin{tabular}{|c|c|c|c|c|c|c|}
\hline \multirow{2}{*}{ RI } & \multicolumn{2}{|c|}{$\begin{array}{l}\lambda_{\text {peak }} \\
{[\mathrm{nm}]}\end{array}$} & \multicolumn{2}{|c|}{$\begin{array}{c}\mathrm{S}_{\lambda} \\
{[\mathrm{nm} / \mathrm{RIU}]}\end{array}$} & \multicolumn{2}{|c|}{$\begin{array}{c}\mathbf{R} \\
{[\mathrm{RIU}]}\end{array}$} \\
\hline & Ag & Au & Ag & Au & Ag & Au \\
\hline 1.28 & 430.6 & 545.9 & 970 & 700 & $\begin{array}{c}1.03 \mathrm{x} \\
10^{-4}\end{array}$ & $\begin{array}{r}1.42 \mathrm{x} \\
10^{-4}\end{array}$ \\
\hline 1.30 & 450.3 & 560.4 & 1180 & 800 & $\begin{array}{c}8.47 \mathrm{x} \\
10^{-5}\end{array}$ & $\begin{array}{c}1.25 \mathrm{x} \\
10^{-4}\end{array}$ \\
\hline 1.32 & 474.6 & 577.9 & 1480 & 1080 & $\begin{array}{c}6.76 \mathrm{x} \\
10^{-5}\end{array}$ & $\begin{array}{c}9.25 \mathrm{x} \\
10^{-5}\end{array}$ \\
\hline 1.34 & 505.1 & 600.8 & 1980 & 1580 & $\begin{array}{c}5.05 \mathrm{x} \\
10^{-5}\end{array}$ & $\begin{array}{c}6.32 \mathrm{x} \\
10^{-5}\end{array}$ \\
\hline 1.36 & 545.5 & 636.1 & 3010 & 2630 & $\begin{array}{c}3.32 \mathrm{x} \\
10^{-5}\end{array}$ & $\begin{array}{r}3.80 \mathrm{x} \\
10^{-5}\end{array}$ \\
\hline
\end{tabular}

\section{Conclusion}

A refractive index SPR sensor based on a D-shaped photonic crystal fiber with a bimetallic layer is analyzed in this work. This approach of a multiplasmonic sensing configuration is not based on coupling between plasmons, but on the generation of two distinct and independent

\footnotetext{
*e-mail: markosdenardi@gmail.br

**e-mail: jweyl@ufpa.br
}

plasmons, with distinct responses with potential interest for multiparameter sensing. Preliminary results of a single parameter (refractive index) show a sensitivity of 3010 $\mathrm{nm} / \mathrm{RIU}$ and a resolution of $3.32 \times 10^{-5} \mathrm{RIU}$.

This study was financed in part by the Coordenação de Aperfeiçoamento de Pessoal de Nível Superior - Brasil (CAPES) - Finance code 001 and Conselho Nacional de Desenvolvimento Cientifico e Tecnologico (CNPq). It was also financed by the ERDF_-European Regional Development Fund through the Operational Program for Competitiveness and Internationalization-COMPETE 2020 Program and by National Funds through the Portuguese funding agency, FCTFundação para a Ciência e a Tecnologia within project "GreenNanoSensing" POCI-01-0145-FEDER-032257.

\section{References}

1. JunJun $\mathrm{Wu}$, Shuguang Li, Xinyu Wang, Min Shi, Xinxing Feng and Yundong Liu, Applied Optics, Vol 57, No 15, 2018.

2. S. Jabbari, B. Dabirmanesh, S. S. Arab, M. Amanlou, and S. Daneshjou, Sens. Actuators B 240,519-527, 2017.

3. Md. Rabiul Hasan, S. Akter, A.A. Rifat, S. Rana, K. Ahmed, R. Ahmed, H. Subbaraman, D. Abbott, Biosens. IEEE Sens. J. 18 (1) (2017) 133-140F. De Lillo, F. Cecconi, G. Lacorata, A. Vulpiani, EPL, 84, 2008.

4. Wang, Yong \& Huang, Qing \& Zhu, Wenjie \& Yang, Minghong \& Lewis, Elfed, Optics Express. 26.1910, 2018.

5. Thenmozhi, Harikesavan \& Mani Rajan, Senthil \& Ahmed, Kawsar, Optik - International Journal for Light and Electron Optics. 180. 264-270. 2019.

6. D. F. Santos, A. Guerreiro, J. M. Baptista, Optik International Journal for Light and Electron Optics. IEEE Sensors Journal, 17, 8, 15, 2017. 